\title{
Sesgos y dificultades en la investigación de los nuevos antipsicóticos ${ }^{1}$
}

RESUMEN: En este trabajo se pone de manifiesto lo limitados y poco generalizables que son los ensayos clínicos controlados aleatorizados con fármacos debido a errores y dificultades de diseño, análisis e interpretación de resultados, los conflictos éticos que plantean y lo sesgada que es la información que nos llega a través de las revistas científicas, todo ello ejemplificado con los nuevos antipsicóticos.

PALABRAS CLAVE: Investigación farmacológica. Ensayo clínico controlado. Sesgos de publicación. Ética. Nuevos antipsicóticos. Antipsicótico atípico.
SUMMARY: We describe the limitations of randomised clinical trials of drugs because of pifalls in methodological design, analysis and interpretation of results, ethical conflicts and publication bias. We analyse trials on new antipsychotics as example of these pitfalls and limitations.

KEY WORDS: Pharmacological investigation. Randomised clinical trial. Publication bias. Ethics. New antipsychotics. Atypical antipsychotic.

\section{"Vosotros, los que gobernáis este mundo poderoso y sus poderosos asuntos con las máquinas de la elocuencia; los que lo calentáis y enfriáis, ablandáis y modificáis para luego volverlo a endurecer según vuestros propósitos [...] y también vosotros, los que sois guiados y llevados como pavos al mercado, con una vara golpedindoos y una caperuza roja en la cabeza, meditad, meditad, os lo ruego." Tristam Shandy, de Laurence Sterne.}

\section{Introducción}

Cómo ser̃alan Healey (1) o Shorther (2), quizás por el poco tiempo transcurrido y la dificultad de acceder a las fuentes, la historia de la psicofarmacologia moderna desde la introducción de la clorpromacina en los años 50 , se relata exclusivamente como la historia de unos heroicos investigadores que con una mezcla de creatividad, intuición, tesón y suerte fueron descubriendo las diferentes moléculas (el mito de la serendepity '). De esta manera, se minimiza el importante papel en su génesis y desarrollo de factores culturales, económicos y políticos, sobre todo la importancia de la industria farmacéutica. Este papel central de la industria farmacéutica en la investigación (en nuestro país contribuye en más del $70 \%$ de la financiación total de la inves- 
tigación biomédica (3) no deja de ser controvertido, ya que no hay que olvidar que el interés principal de toda industria es el beneficio económico y secundariamente los intereses sociales y sanitarios. Mientras que desde la industria farmacéutica ${ }^{3}$ se señala que sin su contribución no se hubiera logrado el desarrollo de nuevos tratamientos por lo costoso de los procesos, desde posiciones más críticas se cuestiona que determinen interesadamente las líneas de investigación en ciencias básicas (y no básicas), la categorización de enfermedades y la propia nosología e incluso los modelos de enfermedad y los sociosanitarios (1), (4). Pero la investigación no sólo esta condicionada por factores económicos, sino también por factores éticos (proteger los derechos de los pacientes en los ensayos clínicos...), políticos (asignación pública en $\mathrm{I}+\mathrm{D}$, normas que regulen la introducción de nuevos fármacos, sistema sanitario...) y culturales (papel asignado por la sociedad a la medicina y la tecnología biomédica...), a su vez todos interrelacionados. Merece la pena tener en cuenta este marco para entender las claves actuales tanto de la investigación en psicofarmacología ( $y$ en general en salud mental) como del debate científico. Negar la influencia de este conflicto de intereses en el contenido, metodología, interpretación y publicación de los ensayos clínicos sirve para alimentar el mito de que cada vez contamos con más y mejores evidencias cientificas sacadas de estudios imparciales de gran calidad metodológica con las que tomar las decisiones clínicas y de política sanitaria (5).

Desde los primitivos ensayos clínicos de Delay con clorpromacina hasta los modernos estudios multicéntricos controlados y aleatorizados ha habido una creciente sofisticación en los métodos y tecnologías de las investigaciones psicofarmacológicas debidos a las exigencias de la comunidad científica. Sin embargo para algunos autores como Healey (1) esta sofisticación sólo ha generado mayor confusión y la impresión de que sólo ha servido para que los investigadores puedan concluir lo que quieran. Paradójicamente en un análisis que Thornley y Adams (6) realizan sobre la calidad de 2000 ensayos clínicos controlados sobre la esquizofrenia en los últimos 50 años encuentran que esta es muy baja y que no mejoraba en el transcurso de los años. Además se añade otro factor de confusión: el problema del contenido, análisis, acceso y divulgación de la información. La actual profusión de ensayos clínicos genera una cantidad ingente de informacion, en ocasiones redundante y contradictoria que es imposible de metabolizar, mientras que otros ensayos no se hacen públicos por determinados problemas o intereses (lo que se ha denominado sesgo de publicación (7). La capacidad de analizar esta información o de acceder a ella ha generado toda una tecnología (revisiones sistemáticas, metaanálisis, bases de datos como la Cochrane...) con el objetivo de crear un cuerpo de evidencia sobre el que basar las decisiones clínicas y que no está exenta de controversia (8) (9).

Un buen ejemplo de la problemática actual de la investigación psicofarmacológica lo encontramos en el caso los nuevos antipsicóticos (NAP). Los NAP han pretendido buscar la eficacia de la clozapina sin el riesgo de agranulocitosis

\footnotetext{
' serendepity en inglés significa "facultad de encontrar algo valioso por casualidad".
} 
de ésta ni los síntomas extrapiramidales (SEP) de los neurolépticos incisivos clásicos. La aparición de los NAP ha generado una avalancha de ensayos (financiados en casi el $100 \%$ por la industria) y publicaciones para respaldar su eficacia y tolerabilidad con las que argumentar su utilización como primera línea de tratamiento (11) (12). Como sefralan Kapur y Remington (13), la esquizofrenia ha sido ignorada durante largo tiempo por la sociedad y las compañías farmacéuticas y cualquier nueva medicación, ya sea realmente mejor o no, se recibe con gran entusiasmo por los profesionales, pacientes y cuidadores. Así los NAP se han convertido en sinónimo de esperanza. Es dificil determinar cuánta de esta esperanza se alimenta de conclusiones científicas y cuánta de elementos de marketing. Para intentar valorar toda esa información y aclarar su relevancia, realizamos una revisión de este tema tomando como punto de partida las revisiones sistemáticas de The Cochrane Library ${ }^{14}$. Aquella revisión nos permitió darnos cuenta de la escasa calidad de los ensayos clínicos y lo poco fundamentada que era la información que transmitían. El objetivo de este trabajo es poner de manifiesto lo limitados y poco generalizables que son los ensayos clínicos controlados (ECC) debido a errores de diseño, análisis e interpretación de resultados, los conflictos éticos que plantean y lo sesgada que es la información que nos llega a través de las revistas científicas, todo ello ejemplificado con los NAP. Con ello no pretendemos un ejercicio de purismo metodológico sino demostrar la dificultad que supone sacar conclusiones de las investigaciones psicofarmacológicas en general $y$, en el caso de los NAP en particular, que estamos muy lejos aún de tener un conocimiento adecuado de cuál es la realidad de su impacto en el tratamiento de las personas con esquizofrenia y en los costes sanitarios.

\section{Diseño de los estudios}

En el caso de los ensayos clínicos sobre tratamientos farmacológicos, el diseño del estudio puede dirigirse más al análisis de la eficacia del fármaco (su efecto terapéutico en condiciones teóricas o ideales) que a la efectividad (efecto terapéutico real en condiciones naturales $y$ habituales) (15). Estos términos no son antónimos sino extremos opuestos de un continuum. La eficacia siempre es mayor que la efectividad porque en condiciones experimentales los resultados son siempre mejores que en condiciones reales. Por eso, es importante conocer qué aspectos del diseño del estudio nos colocan más cerca de la efectividad para poder interpretar los resultados más adecuadamente.

\section{Selección de la muestra. Criterios de inclusión.}

De cara a confirmar la eficacia del fármaco que se está estudiando y la extrapolación de los datos de la investigación a la población general, la selección de la muestra es crucial. Unos criterios de inclusión y exclusión estrictos nos permitirán conocer mejor el efecto terapéutico teórico o ideal del fármaco (eficacia). En el caso de la esquizofrenia, se excluyen de forma habitual a todos aquellos sujetos con comorbilidad o consumo de tóxicos, lo que deja fuera a gran parte de los pacientes. La FDA no contempla en su autorización para la aprobación de medicamentos que estos funcionen en una muestra típica o 
representativa de sujetos, por lo que los estudios de fase III son de eficacia y no de efectividad y sus resultados tienden a ser más positivos que en la situación real. Si el diseño fuera para determinar la efectividad del fármaco, sólo se excluirían a los pacientes por motivos éticos o empíricos y la muestra sería más heterogénea: esto exigiría tamaños muestrales más grandes, pero los resultados serían más generalizables. Si luego se quisiera analizar por subgrupos de determinadas características, la efectividad global sería menor, pero quizás se detectaran subgrupos de pacientes para los que el fármaco fuera mejor que la media y esa información acabaría siendo valiosa.

\section{Tamaño de la muestra.}

Una población de ensayo demasiado pequeña expone al investigador y a los pacientes a dos posibles riesgos: a) pueden ver una ventaja donde de hecho no la hay (error tipo I o a) cuando en realidad es simplemente una cuestión de azar, más aún si se escoge un nivel de significación p bajo; b) pueden no apreciar una ventaja cuando de hecho si la hay (error tipo II o b) porque el ensayo no tiene la potencia estadística suficiente. A menudo se utilizan intencionadamente grupos pequeños con el objeto de no detectar una diferencia. Ello permite llegar a la conclusión inadecuada de que el nuevo fármaco "no es distinto" en eficacia que el fármaco ya existente previamente. Estos son los estudios "me too" (yo también) que han abundado mucho en el campo de los antidepresivos cuando todos han intentado demostrar que son igual de eficaces que la imipramina (16). En el caso de los nuevos antipsicóticos se ve esto con fármacos como la zotepina (17) o quetiapina (18) cuando quieren igualar su eficacia en el tratamiento de los síntomas positivos al haloperidol utilizando para ello ensayos de pequeño tamaño ( $n=90-306$ participantes) o en el caso de la risperidona, olanzapina o remoxipride cuando se quieren igualar a la clozapina (19) con estudios con muestras que oscilan entre 54 y 180 pacientes.

Pero una muestra muy grande también puede inducir a error en la interpretación de los resultados. El problema de estas es que pueden detectar como estadísticamente significativos efectos que pueden no tener relevancia en la práctica clínica, ya que al no ser idénticas las poblaciones y ser la potencia estadística muy elevada, una pequeña diferencia en el instrumento de medida que aparece como significativa no significa nada a efectos prácticos. Esta es la estrategia que se ha utilizado para demostrar diferencias en la eficacia sobre los sintomas negativos de la olanzapina (con un megaensayo de 1996 participantes (20) y risperidona (con otro gran multicéntrico de 1362 (21), cuando la repercusión clínica de estos hallazgos en el funcionamiento diario de los pacientes no está muy clara.

\section{Aleatorización y enmascaramiento}

El proceso de aleatorización es importante para eliminar el sesgo de selección en los ensayos clínicos. Si la selección se realiza mediante ordenadores o tirando una moneda al aire, se evita cualquier preferencia consciente o inconsciente del investigador. En un estudio al respecto, se demostró que las odds ratios se habian sobreestimado en el $41 \%$ en los ensayos en los que no había 
asignación aleatoria y el $30 \%$ cuando dicho método no estaba claramente descrito. De los 14 estudios incluidos en la revisión Cochrane sobre la risperidona (22) y de los 9 de olanzapina (23), que son los mejores disefiados metodológicamente, sólo uno de cada (21) (24) estaban adecuadamente aleatorizados.

La importancia del enmascaramiento o "ciego" se debe a que evita el sesgo del observador. Si nadie conoce cuál es el tratamiento que están recibiendo los pacientes, no es posible que se produzca una sobreestimación sistemática del efecto de un tratamiento en particular. Los estudios sin enmascaramiento sobreestiman el efecto en un $17 \%$. Se ha señalado que cuando en los ensayos clínicos de los nuevos antipsicóticos se usa el haloperidol en el grupo control a dosis fijas y elevadas (por encima de 12 $\mathrm{mg}$ ), la aparición de efectos secundarios de tipo extrapiramidal desvela el "ciego". del estudio.

\section{Dónde y cómo se da el tratamiento.}

La mayor parte de los ensayos clínicos que se realizan sobre los tratamientos antipsicóticos se llevan a cabo en unidades de agudos de hospitales de renombre, con altas dotaciones en recursos e infraestructura. Estas condiciones tan ideales son adecuadas para estudios de eficacia pero poco tienen que ver con la realidad del paciente esquizofrénico que, afortunadamente, pasa la mayor parte de su vida fuera del hospital y es atendido por un médico en un centro ambulatorio bastante menos sofisticado. Por otro lado, el paciente que está ingresado presenta un cuadro clínico diferente al que presenta cuando está en tratamiento ambulatorio y precisa unas dosis antipsicóticas mucho mayores.

Desde un punto de vista más sociocultural, también hay que tener en cuenta que la mayoría de los ensayos clínicos sobre esquizofrenia se realizan en los EE.UU (el $54 \%$ en la revisión de Thornley y Adams (6) cuando solamente el $2 \%$ de la población con esquizofrenia en el mundo vive alli. Cómo se pueden generalizar los resultados a otros contextos sociales, culturales, económicos y políticos (incluida la política sanitaria) es una cuestión que no queda clara.

\section{Tratamientos del grupo control.}

En los estudios de tratamiento farmacológico de la esquizofrenia no parece ético emplear placebo en el grupo control porque se conocen tratamientos eficaces para este trastorno y el retrasar su inicio perjudica al paciente. Sin embargo, es llamativo que apenas hay estudios que comparen los nuevos antipsicóticos con otro antipsicótico que no sea el haloperidol. Muchas veces además se comparan dosis variables del nuevo antipsicótico con dosis fijas y relativamente altas de haloperidol (es decir por encima los 6-12 $\mathrm{mg}$ de haloperidol o equivalente recomendados por ejemplo por la Asociación Americana de Psiquiatria (25). En dos de los estudios comparativos de risperidona con haloperidol de mayor calidad metodológica se emplean dosis variables de risperidona con dosis fijas de $10 \mathrm{mg}$ (21) y $20 \mathrm{mg}$ (26) de haloperidol. En estas condiciones, la incidencia de SEP siempre es mayor en el grupo control como es de esperar $y$, por otro lado, la 
sintomatología negativa secundaria a la medicación antipsicótica (y difícilmente distinguible de los síntomas negativos primarios que son permanentes y resultado de un déficit intrínseco de la enfermedad) también.

En un polémico artículo de Geddes et al. (27) se realiza una revisión sistemática de los ensayos clínicos con los antipsicóticos atípicos y se encuentra que la heterogeneidad de los resultados de los estudios se explica por un efecto de dosis de la medicación control (haloperídol en la mayoría). Cuando se comparaban con dosis adecuadas de haloperidol (menos de $12 \mathrm{mg}$ ) o equivalente no se encontraban beneficios en los antipsicóticos atípicos con relación a su efícacia o tolerancia global, por lo que consideraban que no tenían por qué utilizarse como de primera elección, sino en aquellos pacientes que presentaran SEP intolerables.

\section{Duración del estudio}

Con la perspectiva temporal que da una enfermedad de larga duración como la esquizofrenia, una de las mayores críticas que se hacen a los ensayos clínicos es la duración de los mismos. Predominan los estudios a corto plazo de menos de 6 semanas (el 50\% duraban menos de 6 semanas frente a un $19 \%$ de más de 6 meses en la revisión de Thornley (6). La duración total de la mayor parte de los ensayos clínicos con los nuevos antipsicóticos oscila entre las 4-12 semanas. En este corto periodo de tiempo se pueden sacar algunas conclusiones sobre eficacia y tolerabilidad del fármaco a corto plazo en situaciones de descompensación clínica y en régimen de ingreso, pero en ningún caso se puede analizar el funcionamiento global del paciente, su grado de adaptación social y familiar, adherencia al tratamiento y tampoco parámetros de eficacia, tolerabilidad y respuesta clínica a medio y largo plazo. De los estudios seleccionados por la Cochrane para sus revisiones, sólo una minoría eran disef́os a largo plazo (más de seis meses): dos de risperidona (22), uno de olanzapina (23), dos de ziprasidona (28) y ninguno de quetiapina (18) ni de zotepina (17). Es de esperar que se realicen ensayos clínicos más largos.

\section{Medidas evolutivas. El uso de escalas}

La historia de la evaluación de la esquizofrenia resume en gran medida la propia historia de la nosología psiquiátrica. La utilización de escalas en los ECC es todavía un foco de investigación importante y aunque se han perfeccionado los instrumentos, las dificultades en la cuantificación de una noción tan abstracta como la esquizofrenia continua siendo un objeto de debate. En las últimas décadas ha habido un desarrollo de diferentes aproximaciones a la conceptualización de la esquizofrenia, en cierto sentido competitivas, con el desarrollo de modelos categoriales y dimensionales (esquizofrenia tipo I y II de Crow, síntomas positivos y negativos de Andreasen o el modelo tridimensional de Liddle) (29). La falta de un patrón oro o de variables claves en las que basar el desarrollo de un constructo operacional hace que los instrumentos de evaluación diseñados acaben utilizándose para estudiar la validez del propio modelo que determinó su diseño. Estos problemas reflejan uno mayor: aún no se ha establecido 
definitivamente el tipo de entidad nosológica al que pertenece la esquizofrenia. Es importante señalar esto porque las escalas que se utilizan en los ECC arrastran una validez de concepto (esto es que el agrupamiento de síntomas y signos de la entidad llamada esquizofrenia, son dimensiones psicopatológicas que tienen que ver con un sustrato neurobiológico por determinar) muy limitada y polémica. Es decir, ¿es válido el concepto que estamos midiendo? No es de extrañar por tanto que en el análisis de Thornley y Adams (6) aparezcan hasta 640 instrumentos diferentes de medida.

Las escalas más utilizadas en los ECC para medir los síntomas son la BPRS (Overall y Gorham 1962) (30) que fue diseñada con el objetivo de evaluar la respuesta al tratamiento psicofarmacológico y la PANSS (Kay y cols.) (31) que se elaboró a partir de la anterior para una evaluación del modelo positivo/negativo intentando diferenciar los síntomas negativos primarios y otros síntomas no relacionados con estas dimensiones. Son escalas que lleva un tiempo considerable pasarlas, minuciosas $y$ en las que es muy fácil encontrar diferencias significativas sobre todo cuando se disgregan en las diferentes subescalas. La critica mayor que se hace en las medidas de eficacia de los ECC es la dificultad en la interpretación clínica de las diferencias estadísticas encontradas $y$ el hecho de que las escalas son raramente utilizadas en la practica diaria. Generalmente la eficacia viene definida por una reducción del $20 \%$ al $40 \%$ en la puntuación media de las escalas PANSS o BPRS pero, ¿cómo se traduce en el funcionamiento diario y cómo se puede generalizar una mejoría mayor de un $20 \%$ en la escala BPRS de un paciente ingresado en un hospital americano? Otro problema añadido es la valoración de la gravedad y el cambio. En general a mayor puntuación de una escala hay mayor gravedad, sin embargo no son escalas de intervalo (es decir una puntuación de 60 no quiere decir exactamente que el paciente esté el doble de peor que uno que haya puntuado 30 , pues dependería del análisis de los factores en los que más ha puntuado y en su traducción clínica). Muchas veces los resultados se expresan como una mejoría en la puntuación media de las escalas, siendo muy diferente por ejemplo, que un paciente disminuya 20 puntos desde una puntuación máxima de 80 que si su nivel basal era 60 . Estas especificaciones con frecuencia se omiten en los estudios. En la mayoría de las ocasiones los estudios no utilizan evaluaciones clínicas categoriales sobre los pacientes que han conseguido una mejoría clínica significativa.

En el caso de los síntomas negativos el problema es aún más complejo por la menor validez de este concepto y por las dificultades de las escalas para diferenciar entre los síntomas negativos primarios y los secundarios a la medicación antipsicótica o a los síntomas depresivos (32).

\section{Análisis de los resultados}

1. Expresión de los resultados

Cuando se comienzan los ensayos clínicos controlados aleatorizados con un nuevo fármaco y si no se inician de forma prematura, hay suficientes estudios 
previos que ya rechazan la hipótesis nula de que no hay diferencias entre el grupo control y el grupo tratamiento. El objetivo de ese ensayo es por tanto, intentar estimar con precisión la magnitud del efecto del tratamiento, establecer si este efecto es suficientemente grande para apoyar reivindicaciones de significación clínica o política. Para tal reivindicación, la diferencia estadística es necesaria pero no es suficiente. Por este motivo, aquellos ensayos que cuenten sus resultados sólo en términos de tests estadísticos o valores de $p$ (probabilidad de error) deben ser rechazados (15). Como muchos de los resultados estadísticamente significativos son de escasa importancia clínica 0 práctica y son habituales en estudios potentes con tamafios muestrales grandes, la principal finalidad de un ensayo clínico debería ser calcular la envergadura del efecto terapéutico con sus intervalos de confianza. Los resultados para variables binarias deben expresarse utilizando el riesgo relativo, la reducción del riesgo relativo y la reducción del riesgo absoluto y deben darse los intervalos de confianza para determinar cuán precisos son los resultados. También debe calcularse el número de pacientes que es necesario tratar (NNT) para evitar un episodio. Los resultados para variables continuas deben expresarse mediante diferencia de medias y sus intervalos de confianza.

\section{Análisis de la eficacia y efectividad}

Como hemos sefralado la valoración de la eficacia de los NAP (no digamos de la efectividad) en los ensayos clínicos está limitada, entre otras cosas, por los instrumentos de medida de los que disponemos. Con estos instrumentos de medida de eficacia, los metaanálisis de los ensayos clínicos de los NAP han demostrado que tienen una eficacia comparable con los antipsicóticos convencionales y en algunos de ellos (olanzapina y risperidona) incluso superior, sobre todo en los estudios a corto plazo. Sin embargo en estos casos, cuando se controlan algunas de las variables las diferencias disminuyen (como en el caso de los abandonos que se comentará más adelante) o desaparecen (como en el caso del efecto de la dosis del grupo control en la revisión sistemática de Geddes et al, (27), que no encuentran diferencias en la eficacia en dosis de haloperidol inferiores a $12 \mathrm{mg}$ ).

La supuesta eficacia de los NAP en los síntomas negativos es aún más controvertida. En general, la eficacia de los NAP en los síntomas negativos se ha valorado en estudios en que los pacientes fueron seleccionados para ver su respuesta en los síntomas positivos. En estos pacientes los sintomas negativos parecen responder mejor a los antipsicóticos que los pacientes con estados deficitarios primarios (33). Este tipo de resultados se han presentado para la olanzapina, risperidona, clozapina y zotepina. Como hemos señalado, hay una gran dificultad en diferenciar la sintomatología negativa propiamente dicha de los síntomas negativos secundarios que se asocian a los sintomas positivos, al tratamiento farmacológico y a los síntomas depresivos, y muy pocos estudios se han diseñado para estudiar la mejoría en pacientes con sintomatología principalmente deficitaria. Cualquier conclusión sobre la eficacia de un fármaco 
en esta dimensión debe interpretarse con mucha cautela por los anteriores motivos y porque la dificultad de traducir clínicamente estos hallazgos es aún mayor.

En cuánto a la esquizofrepia resistente, hasta ahora el único antipsicótico que ha demostrado mayor eficacia sobre los antipsicóticos convencionales ha sido la clozapina. La eficacia de los NAP en la esquizofrenia resistente esta aún por demostrar. Existen pocos estudios que evalúen esta hipótesis y debido a la heterogeneidad de los criterios para considerar un paciente como resistente en las revisiones sistemáticas no se pueden establecer conclusiones. Asímismo son necesarios más estudios que comparen los NAP con la clozapina.

Pero el verdadero talón de Aquiles de la valoración de la eficacia es cómo se traducen las diferencias estadísticas encontradas en las puntuaciones de las escalas de síntomas en diferencias clínicas relevantes para el paciente y sus cuidadores. El enfoque de los ensayos hacía la eficacia, hace que muchos autores critiquen la falta de evaluación de cuestiones como la calidad de vida, el funcionamiento social, la utilización de recursos, la satisfacción de los cuidadores, etc., a pesar de que en las conclusiones siempre introduzcan valoraciones sobre la mayor efectividad de los NAP. Para muchos, éste es uno de los puntos cruciales de la investigación con los NAP y reclaman ensayos clínicos "prácticos" (pragmatics trials) en que se valore de forma global los beneficios en la salud y calidad de vida, en cierto sentido complementarios de los de eficacia (34).

Por último no hay que olvidar que los NAP tienen un elevado coste. No existen estudios farmacoeconómicos adecuados que corroboren la hipótesis de una posible ventaja de los NAP en el costeefectividad sobre los fármacos clásicos, pese a la insistencia en el marketing de las compañías fabricantes en la disminución de los costes totales, tanto indirectos como directos. Hasta la fecha la mayoría de las evaluaciones económicas se han basado en estudios retrospectivos de la utilización de recursos médicos obtenidos en muestras relativamente pequeñas y poco generalizables, en estudios prospectivos con pocos pacientes o en modelos de análisis de decisión clínica patrocinados por la industria y con sus propios datos. En estos estudios tampoco se tienen en cuenta variables como la calidad de vida, funcionamiento social, etc.

\section{Análisis de los abandonos}

Es habitual en los ensayos clínicos que prueban los nuevos antipsicóticos que no se contabilicen los resultados de los abandonos al final del estudio, lo que complica aún más las interpretaciones sobre los datos de eficacia antes comentados. Esta práctica carece de rigor porque sólo nos informa si el medicamento nuevo funciona en aquellos pacientes muy cumplidores que finalizan el ensayo y esto impide la generalización de resultados, sobre todo si tenemos en cuenta que los abandonos en estos estudios rondan el $40 \%$. Para evitar esto se sugiere realizar el análisis según la intención de tratar (intention to treat analysis), que 
supone que aquellos que abandonan el grupo de tratamiento o el de control tienen una evolución desfavorable y cuentan así en los resultados finales. Sin embargo el método que se emplea habitualmente en los estudios es la última medida arrastrada, es decir, el último valor que presentó el paciente en cada variable, repetido en las siguientes mediciones hasta el final del estudio. Sin embargo, este método es benévolo con los pacientes que abandonan cuando el tratamiento es ineficaz porque es de esperar que sigan empeorando.

\section{Análisis de los efectos secundarios}

Este es un tema crucial, ya que la más importante justificación para la introducción de los NAP en el mercado ha sido su mejor perfil de efectos secundarios extrapiramidales y su supuesta mejor tolerabilidad. En general se puede decir que los protocolos de recogida de datos de los efectos secundarios son bastante rigurosos y bien diseffados. La crítica viene de la forma en que son analizados y publicados, ya que muchas veces no están estandarizados los procedimientos de publicación lo que hace difícil la comparación de los estudios y los metaanálisis. Por ejemplo muchas veces sólo se comunican aquellos en los que se han encontrado diferencias estadísticas significativas, lo que puede falsear la realidad por la posible existencia de errores tipo I y tipo II. En otras ocasiones sólo se publican aquellos efectos secundarios que aparecen en más de un $10 \%$ de la muestra, con lo que no se informa de efectos secundarios raros pero no menos importantes (agranulocitosis). Los tamaños muestrales pequeños y la duración corta de algunos ensayos (quetiapina) no valoran la aparición de efectos secundarios poco comunes o de aparición tardía como la discinesia tardía. Un caso especialmente llamativo es la forma en como se han presentado los datos referentes a la ganancia de peso, sobre todo con la olanzapina: incompleta, confusa y minimizando su importancia e impacto en los pacientes (35) (36).

\section{Interpretación de los resultados}

Teniendo en cuenta todas las dificultades metodológicas y sesgos que hemos ido señalando las interpretaciones que aparecen en los artículos de los ensayos clínicos, revisiones no sistematizadas $u$ opiniones de expertos deben ser examinadas con mucha cautela. Hay que tener cuidado con el poder de las palabras y las máquinas de la elocuencia. Se debe tener en cuenta si se pueden aplicar los resultados en tu medio, si se han considerado todos los resultados clínicamente importantes y si los beneficios merecen la pena frente a los perjuicios y costes, porque todo esto se enmascara muchas veces en las discusiones y en los resúmenes de los artículos. A menudo, la base para interpretar una supuesta ventaja son los datos intermedios, debido al problema de los abandonos, en lugar de resultados finales que son los que tienen verdaderá importancia clínica. Incluso cuando no se detectan diferencias significativas, es frecuente ver cómo los autores interpretan sus resultados como un rotundo éxito basándose en datos parciales. A menudo se minimizan los resultados negativos (por ejemplo los efectos extrapiramidales de la risperidona o la ganancia de peso con la olanzapina) y se exageran diferencias 
significativas conseguidas gracias a un alto poder estadístico pero de dudosa traducción clínica (no es que nuestros pacientes se resistan a mejorar de su sintomatología negativa como lo hacen los de los ensayos clínicos).

A menudo aparecen conclusiones precipitadas e inferencias ingenuas $y$ lineales no demostradas que luego son repetidas por los estrategas del marketing y que tienen un impacto importante en los clínicos. Merece la pena señalar dos de ellas que con frecuencia se repiten y con las que se sostiene la indicación de los NAP como de primera elección en el tratamiento de los pacientes con esquizofrenia.

La primera se refiere a la relación entre los síntomas extrapiramidales y la adherencia al tratamiento. De los estudios se puede concluir que los NAP en conjunto (aunque con notables diferencias individuales) producen menor incidencia de SEP entre los pacientes y menores tasas de abandono a corto plazo. De este hallazgo se pasa a la conclusión no demostrada de que al producir menos SEP los NAP son mejor tolerados, hay un mayor cumplimiento del tratamiento, se producen menos recaídas y a larga se produce una mejor relación costeefectividad al evitar ingresos o los efectos deteriorantes de la enfermedad. Como señalan Geddes et al. (27) en su estudio esto implicaría que si un paciente tiene efectos secundarios con un tratamiento ya no va a querer tomar otro. Aún más cuando se utilizan dosis adecuadas de haloperidol (y no dosis fijas excesivas por encima de $12 \mathrm{mg}$ como se utilizan en los ensayos) no encuentran ni mayor eficacia ni menor porcentaje de abandonos entre los NAP y el control. Es decir que la menor incidencia de SEP puede estar contrarrestada por otros efectos secundarios importantes como por ejemplo el aumento de peso (que tiene importantes consecuencia en la salud). La adherencia al tratamiento no está determinada únicamente por la aparición de un efecto secundario, sino por un conjunto de factores como el tipo de enfermedad, la personalidad del paciente, la relación terapéutica, el ambiente social, el sistema sanitario, etc. Todavía no existen estudios que evalúen esta supuesta ventaja de los NAP que haya valorado convenientemente la adherencia, la prevención de recaídas y los costes sociosanitarios. Los escasos estudios farmacoeconómicos (y también patrocinados por la industria farmacéutica) se basan en simuladores de gasto que se fundamentan en este tipo de aseveraciones para justificar el uso de los nuevos antipsicóticos (37) (38).

Otra de las conclusiones no demostradas es en relación con la discinesia tardía. La hipótesis de la que se parte es que la menor incidencia de SEP implica un menor riesgo a largo plazo de discinesia tardía (39) (40). Estos datos todavía son preliminares y poco concluyentes y la incidencia de la discinesia tardía de los NAP todavía está por conocerse (27) (35).

\section{El sesgo en la publicación}

A la hora de diseñar ensayos clínicos para valorar intervenciones en la esquizofrenia y demás trastornos psicóticos hay que tener en cuenta que 
se tratan de problemas complejos de larga duración, con gran variabilidad individual, social y cultural y cuyo pronóstico depende de múltiples factores interrelacionados. El primer sesgo se podría decir que es de contenido ya que con mucha diferencia el mayor peso de la investigación recae en los ensayos farmacológicos. En el análisis de Thomley y Adams (1998) (6) de 2000 ensayos clínicos controlados de intervenciones en la esquizofrenia en 50 años encuentran que el $86 \%$ evaluaban la eficacia de los diferentes fármacos por un $8 \%$ de intervenciones psicoterapéuticas y un $9 \%$ sobre programas de cuidado o tratamiento.

El sesgo de publicación se refiere al fenómeno por el cual los estudios de investigación con resultados "positivos" estadísticamente significativos (diferencias estadísticamente significativas a favor del grupo estudiado sobre el control) se publican en mayor medida que los que obtienen resultados "nulos" (sin diferencias estadísticamente significativas) o "negativos" (diferencias estadísticamente significativas a favor del grupo control sobre el estudiado). La consecuencia de este sesgo es que la información de dominio público de las revistas científicas no es representativa de la totalidad de la evidencia investigadora porque los estudios muestran resultados en una sola dirección (41). Sin embargo, parece tan importante saber si un nuevo tratamiento es mejor que los anteriores, como saber si no aporta nada o incluso si es peor que sus predecesores.

Se han descrito distintas fuentes (41) (42) (tabla 1) y tipos de sesgo. El sesgo idiomático consiste en que la investigación realizada en países de habla no inglesa tiende a publicarse en revistas de lengua inglesa si los resultados son positivos, mientras que si los resultados son negativos tienden a publicarse en una revista del idioma de origen (43). Por otro lado existe el sesgo debido a la mayor rapidez en la aceptación de los trabajos con resultados positivos ${ }^{44}$, lo que aumenta su cantidad publicada, así como que tienden a ser publicados en revistas de mayor impacto editorial que se adquieren con más facilidad y están más extendidas (41) (42). Todos estos sesgos se ponen de manifiesto si se hace una búsqueda limitada a MEDLINE. Este método excluye a revistas de menor impacto, a muchas de las que no se publican en inglés por lo que finalmente sólo se recoge el $30-60 \%$ de los ensayos aleatorizados disponibles (41). El sesgo de publicación múltiple o "salami slicing" consiste en publicar un solo estudio con resultados positivos de forma troceada en distintos artículos. Por ejemplo, si es un multicéntrico, se publican los resultados de cada centro de investigación en cada artículo, o si se trata de un estudio prospectivo, se sacan diferentes artículos en distintos momentos del estudio o simplemente se van publicando partes de los resultados (41) (42). Es importante, para ello, ir alterando el orden y la composición de los autores para que parezcan estudios distintos (un ejemplo de esto es cómo de un solo estudio con olanzapina han aparecido publicados más de 80 artículos) (23). Un último sesgo es el de citación que consiste en que los estudios positivos son más propensos a ser citados por otros autores. Se han planteado distintas soluciones (41) (tabla 2) que ayudarían a paliar este problema. 
Los factores que influyen para que un estudio se publique son de los más curiosos y paradójicos. Se publica más, como hemos dicho, los que obtienen resultados "positivos", especialmente aquellos que son ensayos clínicos de muestras pequeñas, no aleatorizados ni controlados, observacionales $\dot{y}$ patrocinados por la industria farmacéutica. La calidad del estudio no influye salvo de forma paradójica: aquellos con resultados nulos o negativos tienen la misma calidad o más que los que obtienen resultados positivos ya que los primeros tendían a emplear en mayor medida la aleatorización y el doble ciego que los segundos (42).

La consecuencia que se deriva de todo esto es que la información que aparece en las revistas cientificas es parcial y muestra resultados en una sola dirección. Los metaanálisis y revisiones que se hacen pueden multiplicar el sesgo porque sus análisis son sólo de parte de los estudios publicados y no de todos los realizados. Esto es particularmente importante porque estos métodos aparecen como precisos, pueden ser muy convincentes y tienen mucho impacto, $y$ lejos de ser la "verdad científicamente demostrada" pueden esconder una gran mentira que forme parte de nuestra práctica clínica y de la que se puede hacer uso desde las multinacionales farmacéuticas y en la política sanitaria.

\section{Conflictos éticos}

Los problemas éticos en la investigación psicofarmacológica los hemos querido presentar de una forma dinámica como conflictos de intereses entre los diferentes agentes participantes.
1- En primer lugar estarían los conflictos de tipo ético que se dan entre el interés del investigador en demostrar sus hipótesis y el deseo de los pacientes de beneficiarse de los nuevos tratamientos sin que en la investigación se arriesgue su salud. La protocolización de los ensayos y la aprobación de los comités de ética de los ensayos es una muestra de la importancia de este tema. En la investigación con pacientes esquizofrénicos existe un debate sobre su capacidad de decisión y la validez del consentimiento informado y la utilización de placebo o periodos de lavado.

2- Conflictos entre el investigador y la industria farmacéutica. La industria es ahora mismo el principal productor de los ECC con los NAP. Es lícito pensar que el investigador se vea sometido a presiones para conseguir los resultados esperados por la compañía, dadas las inversiones millonarias que tienen que hacer con cada nueva molécula. Merton, un sociólogo de la ciencia, considera que el método científico incluye por un lado los métodos técnicos y por otro lado una ética científica en que el desinterés (es decir guiarse en la investigación por la vocación de conocimiento y no por el interés personal o de otros) sería uno de los cuatro imperativos institucionales de la ciencia (45). Este precepto, que es a la vez moral y técnico, puede estar bajo sospecha en el caso de los investigadores de las compañías. Debería extenderse el hábito de hacer públicos los patrocinadores y los compromisos económicos de los firmantes de cada artículo.

3- Conflictos entre los investigadores/ industria y los lectores/sociedad. Este tema tiene ver con los sesgos de publicación 
que hemos comentado antes y que en el análisis de Merton sobre el ethos científico constituye el segundo imperativo institucional que es el comunismo o principio de comunidad ("todos los hallazgos de la ciencia deban ser patrimonio de la sociedad, reduciéndose al mínimo la intervención de criterios de propiedad privada y de secreto, así como todo aquello que pueda ser incompatible con el imperativo de comunicación de los hallazgos"). En primer lugar se plantea si es ético que una determinadas publicaciones sean enviadas y publicadas (las que muestran resultados positivos) y aquellas cuyos resultados no son favorables a la companía no se hagan públicos. La petición de un registro de ECA y la petición a las revistas para que publiquen resultados negativos son intentos de evitar este problema. En segundo lugar está el grave problema ético de la selección de datos, sesgos, ocultación, abusos estadísticos, fragmentación de la información en múltiples estudios, etc.

4- Conflictos entre la industria y la sociedad. Corresponde (o debería corresponder) a la sociedad ejercer un control sobre la investigación a través de las distintas formas de organización política. Los conflictos éticos a este nivel se dan por ejemplo cuando las autoridades sanitarias (ahora ya no es una cuestión nacional sino europea) autorizan la investigación y comercialización de determinados fármacos que muchas veces no aportan nada nuevo a lo ya existentes. Es el caso de los llamados "fármacos me too", ¿es lícito realizar estos pseudoensayos en los que lo que se intenta demostrar es la igualdad de eficacia de un fármaco nuevo, generalmente mucho más caro, frente a uno ya conocido, barato y de probada eficacia? Con la aparición de la olanzapina y la risperidona se ha abierto un nuevo mercado y es posible que en los próximos años asistamos a la aparición de nuevos "neurolépticos me too" que generen más confusión a la existente.

\section{Conclusiones}

1.- La investigación psicofarmacológica y en concreto la investigación sobre los NAP presenta una serie de dificultades metodológicas y de sesgos (unos intencionados y otros no) que cuestionan su calidad y que deben tenerse en cuenta a la hora de leer los artículos, sobre todo aquellos presentados por la industria en sus actividades de marketing. Las principales debilidades de los estudios que ha sido señaladas son la duración breve de los periodos de seguimiento, los criterios de inclusión, las dosis del grupo control, la alta tasa de abandonos, la inadecuada presentación de los efectos secundarios, la falta de datos que valoren la mejoría clínica real y la calidad de vida. Parece importante, en este sentido, apelar al espíritu crítico a la hora de leer artículos y aceptar verdades y saber integrar la escasa evidencia con el juicio clínico y la preferencia de los pacientes.

2- Lo único que queda claro de los estudios aleatorizados y de las revisiones sistemáticas es que actualmente cualquier conclusión (current randomised evidence) sobre la mayor efectividad de los NAP y su mejor tolerancia global es inadecuada y prematura. La aparición de "consensos de expertos" (11) (12) intenta paliar esta falta de evidencia, pero muchos de 
ellos están financiados por la industria farmacéutica y realizados por expertos alejados de la práctica clínica. Parece claro que la elección de un determinado fármaco depende de una serie de factores que varían en las distintas sociedades e individualmente en cada paciente (muchos de ellos se beneficiarán de las ventajas de los NAP en los efectos extrapiramidales).

3- Lamentablemente, los antipsicóticos (ni los antiguos ni los nuevos) no van a curar la esquizofrenia. Parece importante entonces reivindicar y prestar más atención también a investigaciones sobre aproximaciones terapéuticas que mejoren a nuestros pacientes como las psicoterapias individuales, familiares o grupales, entrenamiento en habilidades sociales, rehabilitación cognitiva, intervenciones psicoeducativas... Por otro lado, cabe preguntarse que si sólo se investiga y se publica sobre psicofármacos no acabaremos convirtiéndonos en meros expendedores de medicinas y entonces la planificación asistencial se dirigirá a esta actividad, dejando aún más de lado la creación de otros recursos asistenciales, residenciales y laborales, que son los que probablemente mejorarían de una forma global la evolución de la esquizofrenia.

Tabla 1. Fuentes del sesgo de la publicación

- Investigador: menos tendencia a escribir y que examinen un estudio "negativo" o "nulo".

- Editor: preferencia en la publicación de estudios "positivos" sobre los "nulos" o "negativos".

- Patrocinador: a la industria farmacéutica no le intersa publicar resultados que cuestionen su producto.

Tabla 2. Soluciones al sesgo de publicación

- Métodos estadísticos: funnel plot, rank correlation test...

- Que las editoriales apliquen criterios de calidad del estudio y no de resultados.

- Registro prospectivo de todos los ensayos para saber de ellos aunque no se publiquen.

- Que se publiquen todos los ensayos que se han realizado con resultados nulos o negativos. 


\section{BIBLIOGRAFÍA}

1- Healey DT. "The psychopharmacological era: notes towards a history". Journal of Psychopharmacology 1990; 4(3):152-167.

2- Shorther E. A History of Psychiatry. John Wiley \& Sons, Inc, 1997. Edición Española de J\&C ediciones médicas S.L., 1999.

3- Esteve Soler J. "La industría farmacéutica como impulsora de la I+D en el sector sanitario". Revista de la Administración Sanitaria 1998; 11(7):1-9

4- Tizón JL. "Investigación y docencia en salud mental (I). Reflexiones para el clínico antes de la autoinmolación". Revista de la Asociación Española de Neuropsiquiatria 2001;77:69-96.

5- Chamorro García L. "La alarma sobre la factura de los psicofármacos". Archivos de Psiquiatria 2000; 63(4): 313-316.

6- Thorney B, Adams C. "Content and quality of 2000 controlled trials in schizophrenia over 50 years". BMJ 1998; 317:1181-1184.

7- Gilbody SM, Song F. "Publication bias and the integrity of psychiatry research". Psychological Medicine 2000;30:253-258

8- Desviat M. "La decisión clínica: entre la evidencia y las narraciones". Psiquiatría Pública $2000 ; 12: 119-122$

9- Smith R. "The failings of NICE". BMJ 2000; 321:1363-1364

10- Wahlbeck K, Cheine M, Essali MA. "Clozapine versus typical neuroleptic medication for schizophrenia (Cochrane Review)". En: The Cochrane Library, Issue 4, 1998. Oxford: Update Software

11- Sociedad Española de Psiquiatría. Consenso español de expertos para recomendaciones de actuación en el tratamiento de la esquizofrenia. Madrid: Aula Médica, 2000

12- "Expert consensus guidelines in the treatment of schizophrenia". $J$ of Clinical Psychiatry 1999;6 suppl 11

13- Kapur S, Remington G. "Atypical antipsychotics. (editorial)". BMJ 2000; 321: 1360-1361.

14- Ortiz Lobo A, Mata Ruiz I. "Los nuevos antipsicóticos". Inf Ter Sist Nac Salud 2001;25:1-8

15- Kraemer HC. "Pitfalls of multisite randomized clinical trials of efficacy and effectiveness". Schizophrenia Bulletin 2000;26:533-541

16- Garattini S. "Normas éticas y científicas en los ensayos clínicos con medicamentos". En VV.AA. Estándares éticos y científicos en la investigación. Barcelona: Fundació Victor Grifols i Lucas 1999, pp. 9-27

17- Fenton M, Morris S, De-silva P, Bagnall AM, Cooper SJ, Gammelin G, Leitner M. "Zotepine for schizophrenia (Cochrane Review)." The Cochrane Library 2000, Issue 2, Oxford: Update Software.

18- Srisurapanont M, Disayavanish C, Taimakaew K. "Quetiapine for schizophrenia (Cochrane Review)." The Cochrane Library 1999, Issue 4. Oxford: Update software.

19- Tuunainen A, Gilbody SM. "Newer atypical antipsychotic medication versus clozapine for schizophrenia". (Cochrane Review). The Cochrane Library 1999, Issue 4. Oxford: Update software.

20- Tollefson GD, Beasley CM, Tran PV, Street JS, Krueger JA, Tamura RN, Graffeo KA, Thieme MF. "Olanzapine versus haloperidol in the treatment of schizophrenia, schizoaffective and schizophreniform disorders: results of an international collaborative trial". American Journal of Psychiatry 1997; 154 4 ×7-465. 
DEBATES

21- Peuskens. "Risperidone in the treatment of patients with schizophrenia: a multi-national, multicentre, dobuel blind, paralell-group study versus haloperidol. Risperidone study group" Br. J. Psychiatry 1995; 166; 712-726.

22- Kennedy E, Song F, Hunter R et al. "Risperidone versus typical antipsychotic medication for schizophrenia (Cochrane Review)". En: The Cochrane Library 1998; Issue 3, Oxford: Update Software.

23- Duggan L, Fenton M, Darddenes RM, El-Dosoky A, Indran S. "Olanzapine for schizophrenia (Cochrane Review)". The Cochrane Library 1999; Issue 4. Oxford: Update software.

24- Beasley CM, Hamilton SH, Crawford AM et al. "Olanzapine versus haloperidol: acute phase results of the international double-blind olanzapine rtrial". European Neuropsychopharmacology 1997;7:125-137

25- American Psychiatric Association. Practice guideline for the treatment of patient with schizophrenia. Washinton DC: APA, 1997.

26- Chouinard G., Jones B., Remington G., Bloom D., Addington D., McEwan G.W., Labelle A., Beauclair L., Arnott W. "A Canadian multicenter placebo-controlled study of fied doses of Risperidone and Haloperidol in the treatment of chronic schizofrenic patients". J. Clin Psychomarcol. 1993; 13: 2-40

27- Geddes J, Freemantle N, Harrison P, Bebbinton P. "Atypical antipsychotics in the treatment of schizophrenia: systematic overwiev and metaregression analysis". BMJ 2000; 321:1371-1376.

28- Bagnall AM, Lewis RA, Leitner ML, Kleijnen J. "Ziprasidone for schizophrenia and severe mental illness (Cochrane Review)". En: The Cochrane Library, Issue 2, 2000. Oxford: Update Software.

29- Peralta V, Cuesta MJ. "Clinical models of schizophrenia: a critical approach to competing conceptions". Psychopatology 2000;33:252-258

30- Overall JE, Gorham DR. "Brief Psychiatric Rating Scale". Psychological Reports 1962; 10:799-812.

31- Kay SR, Fiszbein S, Opler LA. "The positive and negative syndrome scale for schizophrenics". Schizophrenia Bull 1987; 13: 261-276.

32- Carpenter WT. "The treatment of negative symptoms:pharmacological and methodological issues". Britis Journal of Psychiatry 1996; 168 (suppl. 29): 17-22.

33- Fleischhacker WW, Hummer M. "Drug treatment of schizophrenia in the 1990s." Drugs 1997; 53(6): 915-929.

34- Roland M, Torgerson DJ. "What are pragmatic trials?". BMJ 1998; 316:285.

35- Barnes TRE, McPhillips MA. "Critical analysis and comparison of the side-effect and safety profiles of the new antipsychotics". Br J Psychiatry 1999;174:34-43.

36- Allison DB, Mentore JL, Heo M et al. "Antipsychotic-induced weight gain: a comprehensive research synthesis". Am J Psychiatry 1999;156:1686-1696.

37- Manskopf JA et al. "Annual health outcomes and treatment cost for schizophrenia populations". J Clin Psychiatry 1999;60(supl 19):14-19

38- Tunis SL et al. "Changes in perceived health and functioning as a cost-effectiveness measure for olanzapine versus haloperidol treatment of schizophrenia" J Clin Psychiatry 1999;60(supl 19):38-45

39- Beasley CM, Dellva MA, Tamura RN, Morgenstern H, Glazer WM, Ferguson K et al. "Randomised double-blind comparision of the incidence of tardive dyskinesia in patients with schizophrenia during long term treatment with olanzapine and haloperidol". $\mathrm{Br} J$ Psychiatry 1999; 74:23-30. 
40- Beasley CM. "Olanzapine and tardive dyskinesia”. Br J Psychiatry 1999; 175: 392

41- Gilbody SM, Song F, Eastwood AJ, Sutton A. "The causes, consequences and detection of publication bias in psychiatry". Acta Psychiatr Scand 2000;102:241-249

42- Easterbrook PJ, Berlin JA, Gopalan R, Matthews DR. "Publication bias in clinical research". The Lancet 1991;337:867-87

43- Egger M, Zellweger-Zähner T, Schneider M, Junker C, Lengeler C, Antes G. "Language bias in randomised trials published in English and German". The Lancet 1997;350:326-329

44- Stern JM, Simes RJ. "Publication bias: evidence of delayed publication in a cohort study of clinical research projects”. BMJ 1997;315:640-5

45- Merton RK. "Los imperativos institucionales de la ciencia". En B. Barnes. Estudios sobre sociologia de la ciencia. Madrid, Alianza, 1980

Fecha de recpeción: $20 / 6 / 01$

*Psiquiatras. Centro de Salud Mental de Salamanca. Área 2. Madrid

Correspondencia:

Iván de la Mata Ruiz.

Centro de Salud Mental de Salamanca.

C/ O'Donnell, 55

28009-Madrid 